\title{
A study on unpredictable shallow water channel behavior by various multipath patterns and experimental data analysis
}

\author{
Sumithra G* and Meganathan D \\ Department of Electronics Engineering, Madras Institute of Technology, Anna University, Chrompet, Chennai - 600044, India \\ *Corresponding Author : gsumithra.rajkumar@gmail.com
}

Submitted :04/02/2020

Revised : :10/03/2021

Accepted : 19/03/2021

\begin{abstract}
In shallow water applications, multipath signal propagation is a major concern for robust communication. Multipath signal propagation is not explicitly seen in the channel, even though it is a main contributor for signal degradation. This fact motivated us to simulate multipath patterns to understand its influence in short-range communication. In this paper, a three-path signal propagation model is presented, where, besides the line of sight (LOS) signal, other two non-line of sight (NLOS) signals contact any point of channel boundary to reach the receiver. In simulation, the combination of eight possible multipath patterns is converged to estimate the received signal. A source fixed in water-tank periodically transmits low frequency acoustic signals $1 \mathrm{kHz}$ and $1.5 \mathrm{kHz}$ to the channel, and the receiver records them. The experiment was repeated for various input signal strengths. It has been observed that the simulation results coincide with the measured values. The good reception is noticed for signal frequency 1 $\mathrm{kHz}$ at $2.5 \mathrm{~m}$ and $1.5 \mathrm{kHz}$ at $1.2 \mathrm{~m}$. This study identifies the optimal signal strength for better signal reception in short range, which drives to the establishment of high-quality communication in shallow water.
\end{abstract}

Keywords: Multipath propagation; Shallow water communication; Acoustic channel; Channel boundary.

\section{INTRODUCTION}

In shallow water wireless surveillance applications, like unmanned patrolling in harbor or secured places around nuclear reactors, a robust communication system and network is required. Information rich signals gathered from those environments are transmitted continuously to nearby data collection center for further analysis. For shallow water acoustic communication (Van Duc Nguyen et al., 2019; Tri Budi Santoso et al., 2016) system design, knowledge about environment (Stojanovic et al., 2009) is essential to devise channel model. Experimental results confirm that the shallow water acoustic channel is highly time-varying (Kaddaouri et al., 2018) and does not necessarily follow a Rayleigh distribution (Hovannes Kulhandjian et al., 2014) as in terrestrial communication.

Though we find similar involvement of fundamental parameters in the analysis of wireless communication system design for air (Landolsi et al., 2016) and water, at the outset both environments are different. However, they have a common concern for signal fading due to multipath propagation. The acoustic signal propagation is influenced by location dependent physical (Umair Mujtaba Qureshi et al., 2016; Fisher et al., 1977) and chemical properties of 
water. The signal degradation in water is not only associated with the propagation distance, depth, temperature (Stojanovic, 2006), but also concerned with several other factors like noise (Urick, 1984) due to marine habitats, man-made activities, and quakes or tremors in ocean floor. Since, the medium characteristics vary, mapping of research methodology adopted in terrestrial to underwater may not end up giving identical results.

Usually, shallow water channel is bounded by top water surface and bottom seabed. The propagation delay profile, scattering function, doppler spread, and correlation coefficient along with the fundamental parameters contribute to (Peterson et al., 2013) envisage channel model. These channel measurements (Chitre, 2007) are vital to realize the model. Breaking the monotonous approach, the chaotic motion (Virovlyansky, 2008) of a ray path was investigated in a deep-water acoustic waveguide. Exhaustive research on multipath signal propagation is required for shallow water applications to work more comfortably in real time conditions. Numerous promising possibilities of travel pattern for transmitted signal to reach the receiver need to be highlighted to explore and visualize multipath propagation effects. Their results contribute towards precise channel model developed for shallow waters.

In the proposed three-path model, besides LOS path, other multipaths contact channel boundary and reach the receiver. Eight different multipath patterns are simulated, and the unrealistic options of multipath travel are ignored. The simulation gives idea on the probable multipath signal propagation patterns in shallow water channel that are not seen explicitly in real time. And the multipath patterns for good signal reception are identified. The shallow water experiment was performed in the laboratory to validate the simulated results. The low frequency acoustic signals of various signal strengths are periodically transmitted in water tank and signals are recorded. For various transmission frequency and range, experiment results are compared with simulated outcomes. The theoretical predictions of certain multipath pattern for signal propagation closely match the channel measurements and signal of lower input signal strengths are better received compared to higher signal strengths. Overall unpredictability is viewed in received signal for these multipath patterns when operated at varied frequency, transmission range and input signal strength and experiment results confirm this. This study exposes changeable behavior of the tough shallow water channel and our analysis display the necessity to revisit generalized channel model for shallow water channel based on multipath propagation.

\section{SIGNAL TRANSMISSION MODEL IN SHALLOW WATER CHANNEL}

This section presents the background for multipath signal propagation in shallow water environment. In underwater acoustic communication, signal mass tries to travel against the medium stiffness by the common occurrence of multipath signal propagation. During propagation, the signal interacts with medium by way of reflection, scattering resulting in signal attenuation. Also, signal spreads as wave front moves outward from the source (Yang, 2012), since same amount of acoustic signal energy cover the ever-increasing area and depends on the propagation distance. At shorter distances from the source, increasing surface area is represented as surface of a sphere. Spreading loss causes signal to decay at a rate of $R^{-2}$, where ' $R$ ' is the transmission range in 'meter' from source. As the sound wave propagates under water, due to viscous friction and ionic relaxation, energy is lost in the form of heat. Absorption loss (Flatte, 2010) is the conversion of energy in propagating signal to heat and it increases with frequency (David Brady et al.,1998). Thorp's model (Thorp,1967) presents the absorption coefficient. Altogether, signal gets attenuated by spreading and absorption when acoustic signal crosses the channel with the inclusion of underwater noises such as noises are ambient noise, receiver noise, equipment assembly noise and so on (Stojanovic et al., 2016). Ambient noise is related to many factors like location, weather conditions (Parastoo Qarabaqi et al., 2013), etc. 


\section{Multipath Model}

Multipath occurs when a signal takes two or more paths from transmitting antenna to receiving antenna other than the LOS. This mechanism of multipath formation is different in deep and shallow water. It is particularly severe in shallow water communication where strong surface and bottom reflections occur in the sea. Significant amount of signal loss happens due to volume attenuation, bottom loss, scattering and reflection (Alenka ,2011; Wang et al.,2019). Multipath depiction in Figure 1 displays the shallow water channel bounded by top water surface and sea bottom guiding the trapped sound energy to propagate between projector (transmitter) and hydrophone (receiver). From the three-path geometry for multipath model, received signal strength is anticipated for different multipath interaction of NLOS signals with channel boundary.

Signal interaction displayed in Figure 2 (a) shows that one path interacts with channel top at ' $\mathrm{R} / 2$ ' $\mathrm{m}$ distance, and another path interacts with channel bottom at ' $\mathrm{R} / 2$ ' $\mathrm{m}$ distance to reach the receiver. These two paths along with direct path, which is close to transmission range constitutes the three-path model. Similarly, interaction of multipath signals at different locations with channel boundary is displayed in Figure 2 (b)-(h). Multipath signals may interact with any of the points P1, P2, P3, P4, P5 of the channel boundary either top or bottom as shown in Figure 3 in real time scenarios.

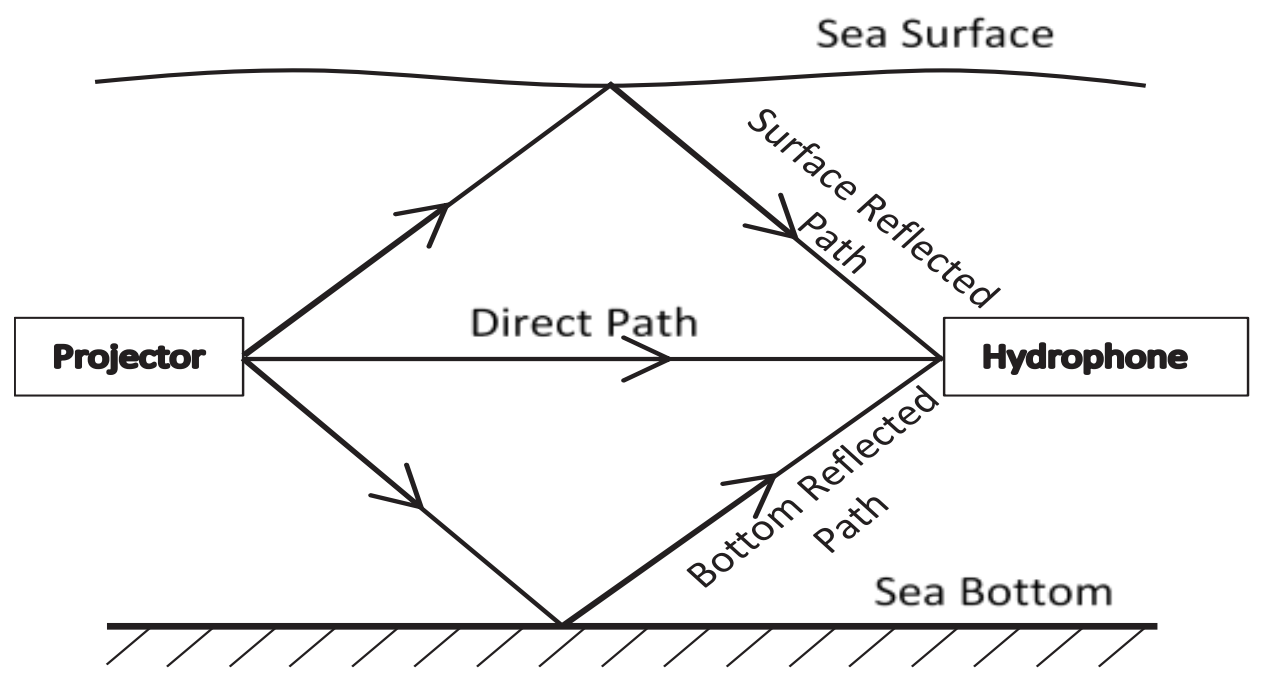

Figure 1. Multipath in Shallow Water Channel.

Interaction of NLOS Multipath signal with Channel boundary

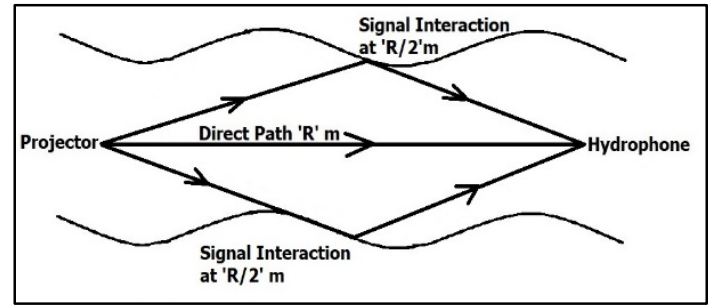

(a)

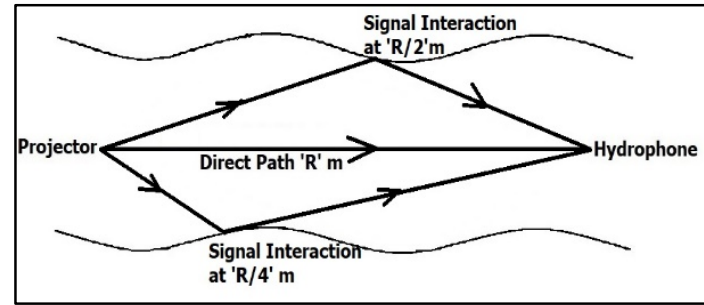

(b) 


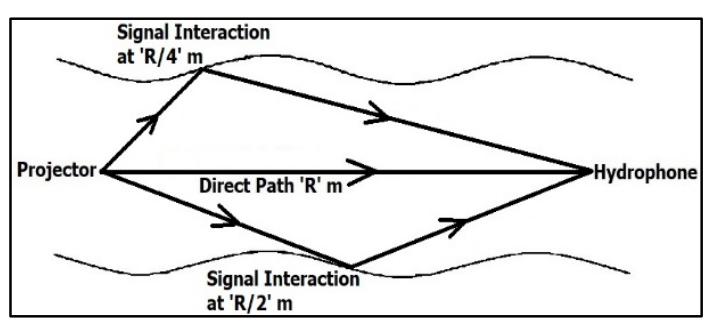

(c)

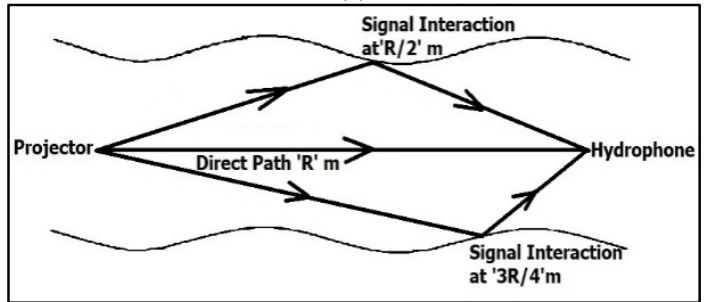

(e)

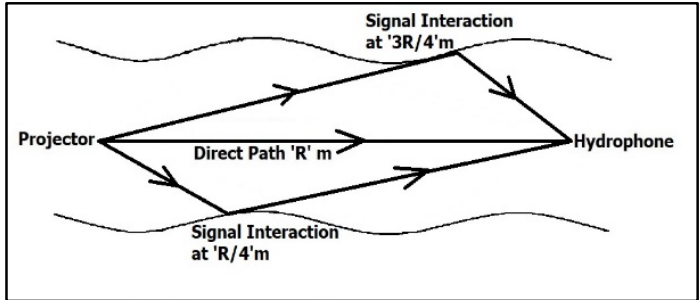

(g)

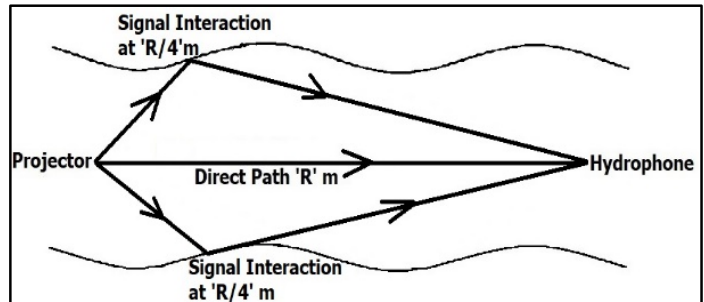

(d)

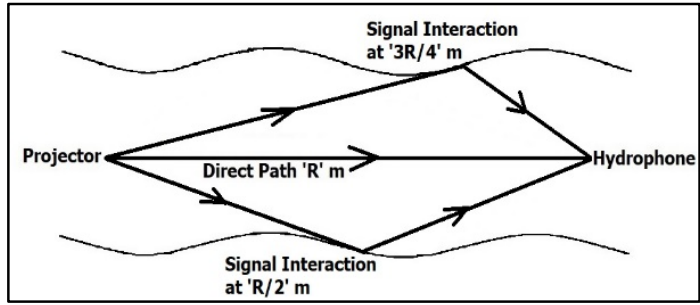

(f)

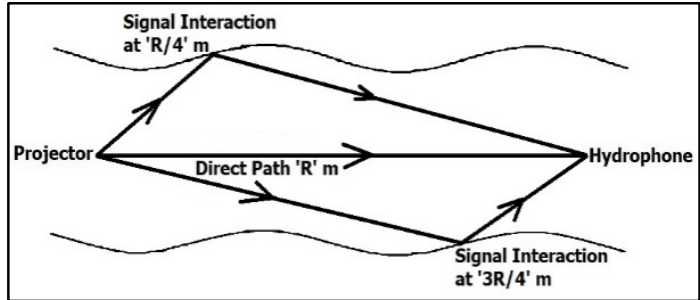

(h)

Figure 2. Multipath Signal Interaction with Channel Boundary.

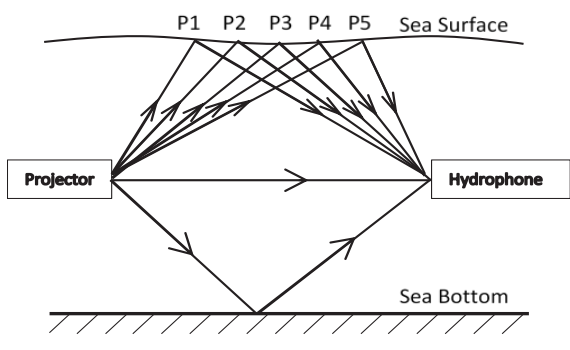

(a)

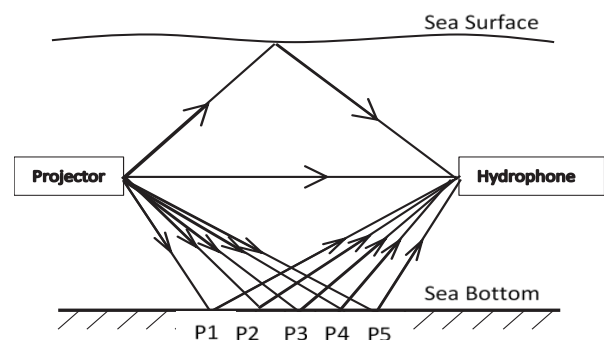

(b)

Figure 3. Signal Hitting Channel Boundary. (a) Top Sea Surface. (b) Bottom Sea Surface.

\section{Estimation of Received Signal Strength}

The signal strength at the receiver from multipath signals arrived by the three-path model for signal propagation in shallow water channel is evaluated. Geometry of multipath pattern for signal transmission from projector $(\mathrm{P})$ to hydrophone (S) is displayed in Figure 4. Direct path is the shortest path which is approximately equal to transmission range, ' $R$ ' $m$. By taking account of multipath interaction with channel boundary, total distance traveled by multipath 
is calculated. Direction of signal travel of other two paths in the medium are identified by angle of projections, $\Theta_{1}$ and $\Theta_{2}$, when signal is released by the projector as shown in Figure 4. With angle of arrival or grazing angle, $\Theta_{3}$ and $\Theta_{4}$, multipath signal reaches the hydrophone. Here path length for the signal reflected from top sea surface is calculated by Equations 1-5. Similarly, path length for the signal reflected from sea bottom is calculated and presented by equation 6.

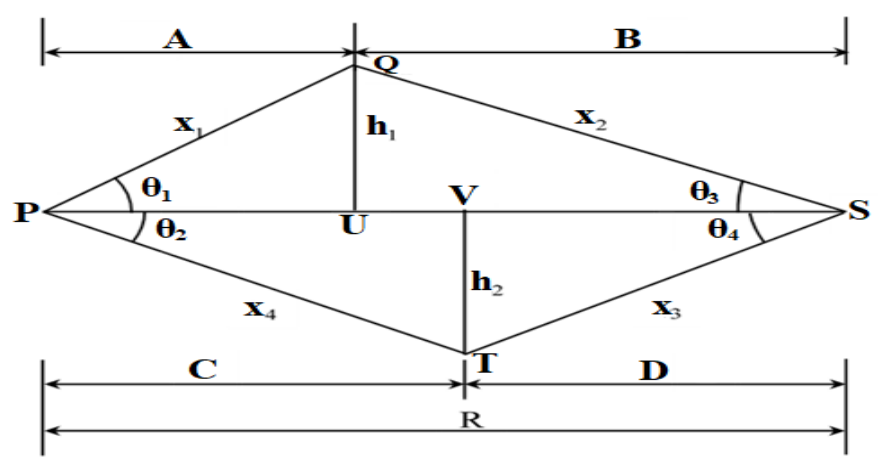

Figure 4. Multipath Geometry in Shallow Water Channel.

$$
\begin{gathered}
x_{1}=\frac{h_{1}}{\sin \left(\theta_{1}\right)} \\
A=\sqrt{x_{1}^{2}-h_{1}^{2}} \\
\mathrm{~B}=\mathrm{R}-\mathrm{A} \\
x_{2}=\sqrt{B^{2}+h_{1}^{2}} \\
L_{\text {TOP }}=x_{1}+x_{2} \\
L_{\text {ВОтTOM }}=x_{3}+x_{4} \\
t_{0}=\frac{R}{C_{0}} \\
t_{1}=\frac{L_{T O P}}{C_{0}} \\
t_{2}=\frac{L_{\text {BOTTOM }}}{C_{0}}
\end{gathered}
$$

The nominal speed of sound $\mathrm{C}_{0}$ in water is $1500 \mathrm{~m} / \mathrm{s}$, and acoustic signal follows isovelocity since the channel is shallow. 
The time delays arrived from equations 7-9 are used to frame the received signal from multiple paths. With time delay of $t_{0}$ from equation 7 , the signal received by direct path from projector to hydrophone, $V_{D}(t)$ is referred in equation 10. Likewise, signal reflected from channel top boundary and channel bottom boundary with their corresponding time delays of $t_{1}$ and $t_{2}$ are represented by $V_{R, 1}(t), V_{R, 2}(t)$ in equations 11-12, respectively. Here, temperature and pressure are assumed constant similar as sound speed in shallow waters.

$$
\begin{gathered}
V_{D}(t)=\cos \left(\omega_{0}\left(t-\tau_{0}\right)\right) \\
V_{R, 1}(t)=\cos \left[\omega_{0}\left(t-\tau_{1}\right)\right] \\
V_{R, 2}(t)=\cos \left[\omega_{0}\left(t-\tau_{2}\right)\right]
\end{gathered}
$$

where $\omega_{0}$ is the frequency. All the delayed version of input signal are linearly added for received signal. The general expression for multipath response for signal propagation in underwater acoustic channel under time-invariant condition is given by Eq. (13),

$$
p(\tau)=\sum_{l} p_{l} \delta\left(\tau-\tau_{l}\right)
$$

where " $\mathrm{p}_{1}$ " is gain of $l^{\text {th }}$ propagation path ( $l$ refers to the path index), propagation delay $\tau_{1}$ is associated with $l^{\text {th }}$ path and $\delta($.$) is dirac delta function. Corresponding to this model is a baseband channel whose impulse response with$ respect to centre frequency $f_{c}$ is given by Eq. (14),

$$
h(\tau)=\sum_{l} h_{l} \delta\left(\tau-\tau_{l}\right)
$$

where, $h_{l}=e^{-j 2 \pi f_{c} \tau_{l}}$ is the equivalent baseband response of the $l^{\text {th }}$ path. With these fundamental channel model expressions, the simulation work based on different multipath travel pattern is exercised.

\section{Simulation of Multipath Signal - Channel Boundary Interaction}

This paper works to bring out the ideal channel response for time-invariant shallow water channel. The multipath time delay influence on the received signal is included in the simulation avoiding distortion or disturbance in signal strength due to signal reflection at channel boundaries. The potential multipath signal interaction points with channel boundary are simulated and their results are reported here. Input projector signal with peak to peak input signal voltages of $1 \mathrm{~V}, 1.5 \mathrm{~V}, 2 \mathrm{~V}$, are generated using MATLAB software.

Simulation focuses the performance of different channel boundary interaction patterns in a multipath scenario to unveil the promising probable multipath patterns. Abiding the fundamental geometry, for projector signal to reach hydrophone, tentatively the first signal interaction with channel boundary can happen $(\mathrm{R} / 4)^{\text {th }}$ distance and not beyond $(3 \mathrm{R} / 4)^{\text {th }}$ distance. Following this distance limits on channel boundary- signal interaction by NLOS signals, eight multipath signal channel boundary interaction patterns, $(\mathrm{R} / 2, \mathrm{R} / 4),(\mathrm{R} / 2,3 \mathrm{R} / 4)(\mathrm{R} / 4, \mathrm{R} / 2)(\mathrm{R} / 4, \mathrm{R} / 4)(\mathrm{R} / 4,3 \mathrm{R} / 4)$, $(3 \mathrm{R} / 4, \mathrm{R} / 2),(3 \mathrm{R} / 4, \mathrm{R} / 4),(3 \mathrm{R} / 4,3 \mathrm{R} / 4)$ are simulated. Time delays infused by equations $7-9$ for direct $\mathrm{LOS}$ path and other two NLOS signals interacting with channel boundary are considered in the simulation. Entire multipath simulation is taken up for transmission ranges, $1.2 \mathrm{~m}$ and $2.5 \mathrm{~m}$ and transmission frequencies $1 \mathrm{kHz}$ and1.5 kHz and procedure is repeated for various input signal strengths. For transmission range of $2.5 \mathrm{~m}$ and $1 \mathrm{kHz}$ transmit signal, simulation results are presented in Table1. And, Table 2 presents the received signal strength for the second transmission frequency of $1.5 \mathrm{kHz}$. The simulation is repeated for another transmission range of $1.2 \mathrm{~m}$ and corresponding results are posted in Table 3 and Table 4 . 
Table 1: Signal Received at Hydrophone for $\mathrm{f}: 1 \mathrm{kHz}-$ Range $=2.5 \mathrm{~m}$.

\begin{tabular}{|c|c|c|c|c|c|c|c|c|c|c|}
\hline \multirow{2}{*}{$\begin{array}{c}\mathrm{I} / \mathrm{P} \\
(\mathrm{V})\end{array}$} & $\begin{array}{c}\mathrm{PRx} \\
(\mathrm{mV})\end{array}$ & $\begin{array}{c}\mathrm{R} / 2, \\
\mathrm{R} / 2\end{array}$ & $\begin{array}{c}\mathrm{R} / 2, \\
\mathrm{R} / 4\end{array}$ & $\begin{array}{c}\mathrm{R} / 4, \\
\mathrm{R} / 2\end{array}$ & $\begin{array}{c}\mathrm{R} / 4, \\
\mathrm{R} / 4\end{array}$ & $\begin{array}{c}\mathrm{R} / 2, \\
3 \mathrm{R} / 4\end{array}$ & $\begin{array}{c}3 \mathrm{R} / 4, \\
\mathrm{R} / 2\end{array}$ & $\begin{array}{c}3 \mathrm{R} / 4, \\
\mathrm{R} / 4\end{array}$ & $\begin{array}{c}\mathrm{R} / 4, \\
3 \mathrm{R} / 4\end{array}$ & $\begin{array}{c}\text { Average of } \\
\mathrm{RX}(\mathrm{mV})\end{array}$ \\
\hline 1 & 353.5 & 97.5 & 91.8 & 97.6 & 91.8 & 91.8 & 97.5 & 91.8 & 91.8 & 93.95 \\
\hline 1.5 & 530.3 & 43.9 & 55.1 & 58.5 & 55.1 & 55.1 & 43.9 & 41.3 & 41.3 & 49.28 \\
\hline 2 & 707.1 & 19.5 & 18.4 & 19.5 & 18.4 & 18.4 & 19.5 & 18.4 & 18.4 & 18.81 \\
\hline
\end{tabular}

Table 2: Signal Received at Hydrophone for f: $1.5 \mathrm{kHz}-$ Range $=2.5 \mathrm{~m}$.

\begin{tabular}{|c|c|c|c|c|c|c|c|c|c|c|}
\hline \multirow{2}{*}{$\begin{array}{c}\mathrm{I} / \mathrm{P} \\
(\mathrm{V})\end{array}$} & \multirow{2}{*}{$\begin{array}{c}\mathrm{PRx} \\
(\mathrm{mV})\end{array}$} & $\begin{array}{c}\mathrm{R} / 2, \\
\mathrm{R} / 2\end{array}$ & $\begin{array}{c}\mathrm{R} / 2, \\
\mathrm{R} / 4\end{array}$ & $\begin{array}{c}\mathrm{R} / 4, \\
\mathrm{R} / 2\end{array}$ & $\begin{array}{c}\mathrm{R} / 4, \\
\mathrm{R} / 4\end{array}$ & $\begin{array}{c}\mathrm{R} / 2, \\
3 \mathrm{R} / 4\end{array}$ & $\begin{array}{c}3 \mathrm{R} / 4, \\
\mathrm{R} / 2\end{array}$ & $\begin{array}{c}3 \mathrm{R} / 4, \\
\mathrm{R} / 4\end{array}$ & $\begin{array}{c}\mathrm{R} / 4, \\
3 \mathrm{R} / 4\end{array}$ & $\begin{array}{c}\text { Average of } \\
\mathrm{RX}(\mathrm{mV})\end{array}$ \\
\hline 1 & 353.5 & 34.9 & 30.2 & 34.9 & 37.8 & 37.8 & 34.9 & 37.8 & 37.8 & 35.76 \\
\hline 1.5 & 530.3 & 26.2 & 34.0 & 39.3 & 34.0 & 34.0 & 39.3 & 34.0 & 34.0 & 34.35 \\
\hline 2 & 707.1 & 52.4 & 45.3 & 52.4 & 45.3 & 45.3 & 52.4 & 45.3 & 45.3 & 47.96 \\
\hline
\end{tabular}

Table 3: Signal Received at Hydrophone for $\mathrm{f}: 1 \mathrm{kHz}-$ Range $=1.2 \mathrm{~m}$

\begin{tabular}{|c|c|c|c|c|c|c|c|c|c|c|}
\hline \multirow{2}{*}{$\begin{array}{c}\text { I/P } \\
(\mathrm{V})\end{array}$} & $\begin{array}{c}\mathrm{PRx} \\
(\mathrm{mV})\end{array}$ & $\begin{array}{c}\mathrm{R} / 2, \\
\mathrm{R} / 2\end{array}$ & $\begin{array}{c}\mathrm{R} / 2, \\
\mathrm{R} / 4\end{array}$ & $\begin{array}{c}\mathrm{R} / 4, \\
\mathrm{R} / 2\end{array}$ & $\begin{array}{c}\mathrm{R} / 4, \\
\mathrm{R} / 4\end{array}$ & $\begin{array}{c}\mathrm{R} / 2, \\
3 \mathrm{R} / 4\end{array}$ & $\begin{array}{c}3 \mathrm{R} / 4, \\
\mathrm{R} / 2\end{array}$ & $\begin{array}{c}3 \mathrm{R} / 4, \\
\mathrm{R} / 4\end{array}$ & $\begin{array}{c}\mathrm{R} / 4, \\
3 \mathrm{R} / 4\end{array}$ & $\begin{array}{c}\text { Average of } \\
\mathrm{RX}(\mathrm{mV})\end{array}$ \\
\hline 1 & 353.5 & 50.7 & 50.7 & 47.0 & 47.0 & 50.7 & 47.0 & 47.0 & 47.0 & 48.39 \\
\hline 1.5 & 530.3 & 76.1 & 76.1 & 79.3 & 79.3 & 76.1 & 79.3 & 79.3 & 79.3 & 78.10 \\
\hline 2 & 707.1 & 38.0 & 38.0 & 35.3 & 35.3 & 38.0 & 35.3 & 35.3 & 35.3 & 36.31 \\
\hline
\end{tabular}

Table 4: Signal Received at Hydrophone for $\mathrm{f}: 1.5 \mathrm{kHz}-$ Range $=1.2 \mathrm{~m}$

\begin{tabular}{|c|c|c|c|c|c|c|c|c|c|c|}
\hline \multirow{2}{*}{$\mathrm{I} / \mathrm{P}(\mathrm{V})$} & \multirow{2}{*}{$\begin{array}{l}\text { PRx } \\
(\mathrm{mV})\end{array}$} & \multicolumn{8}{|c|}{ RX (Received Signal Strength in $\mathrm{mV}$ ) for various multipath patterns } & \multirow{2}{*}{$\begin{array}{c}\text { Average of } \\
\text { RX (mV) }\end{array}$} \\
\hline & & $\begin{array}{l}\mathrm{R} / 2, \\
\mathrm{R} / 2\end{array}$ & $\begin{array}{l}\mathrm{R} / 2 \\
\mathrm{R} / 4\end{array}$ & $\begin{array}{l}\mathrm{R} / 4, \\
\mathrm{R} / 2\end{array}$ & $\begin{array}{l}\mathrm{R} / 4, \\
\mathrm{R} / 4\end{array}$ & $\begin{array}{l}\mathrm{R} / 2, \\
3 \mathrm{R} / 4\end{array}$ & $\begin{array}{c}3 \mathrm{R} / 4, \\
\mathrm{R} / 2\end{array}$ & $\begin{array}{c}3 \mathrm{R} / 4 \\
\mathrm{R} / 4\end{array}$ & $\begin{array}{l}\mathrm{R} / 4, \\
3 \mathrm{R} / 4\end{array}$ & \\
\hline 1 & 353.5 & 95.5 & 95.5 & 95.5 & 95.5 & 95.5 & 95.5 & 95.5 & 95.5 & 95.5 \\
\hline 1.5 & 530.3 & 53.0 & 53.0 & 53.0 & 53.0 & 53.0 & 53.0 & 53.0 & 53.0 & 53.0 \\
\hline 2 & 707.1 & 56.6 & 56.6 & 56.6 & 56.6 & 56.6 & 56.6 & 56.6 & 56.6 & 56.6 \\
\hline
\end{tabular}

$R$ - Transmission Range; I/P - $V_{P P}$ of Input Signal; PRx - $V_{\text {rms }}$ of Projector Signal; 
In all the above tabulations average received signal strength calculated based on eight combination patterns of three path multipath signal transmission model is mentioned and they are compared with experiment findings in the discussion section. Multipath pattern that offer maximum received signal strength are highlighted in the above tables reveals the dominance of certain signal interaction patterns with channel boundary. In Table 4 there is no particular pattern noticed that offers maximum received signal. This shows the existence of inconsistency in multipath signal reception conveying that the actual received signal need not be from the best multipath pattern. Another important observation from the average received signals expose lower input signal strength offer good signal reception.

\section{SHALLOW WATER EXPERIMMNT}

This section presents the details of experiment setup and describe the procedure in conducting the shallow water experiment.

\section{Experimental Setup}

To perform shallow water experiments, an acrylic under water test bed (UTB) with dimensions of UTB is $3 \mathrm{~m} \times$ $2 \mathrm{~m} \times 2 \mathrm{~m}$ kept in the laboratory is used and is shown in Figure 5(a). Experimental setup in the laboratory is displayed in Figure 5(b). Here, source and receiver involved in underwater communication are understood as projector and hydrophone. Source and receiver are $12 \mathrm{~W}$ USB powered, $60 \mathrm{~Hz}-15 \mathrm{kHz}$ frequency response speaker and a microphone with sensitivity of - $46.0206 \mathrm{~dB}$ re $1 \mathrm{~V} / \mathrm{Pa}(5 \mathrm{mV} / \mathrm{Pa})$. Source and receiver are deployed at $60 \mathrm{~cm}$ from tank side walls on both ends and water depth is about $1 \mathrm{~m}$.

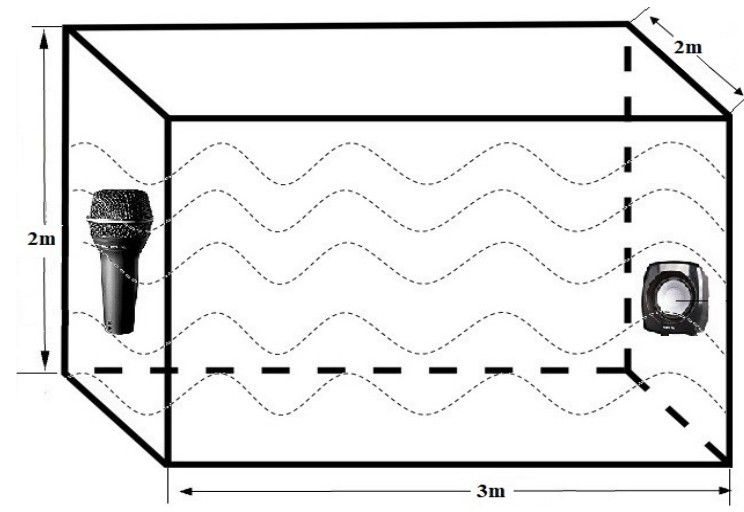

(a)

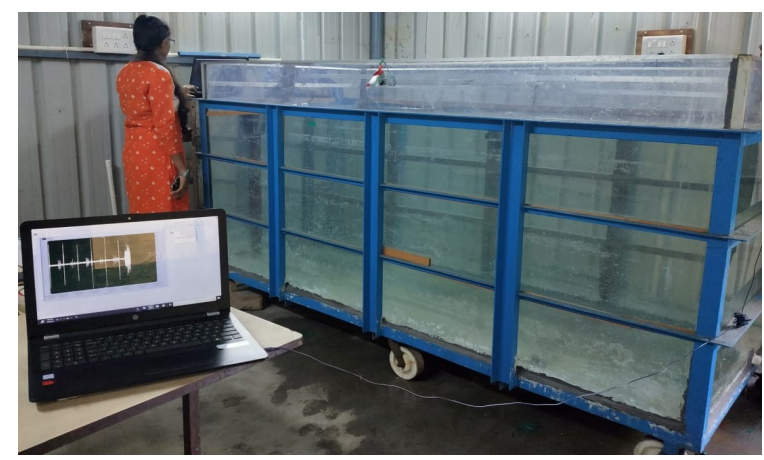

(b)

Figure 5. (a) Underwater Test Bed. (b) Experimental setup in Laboratory.

\section{Measurement Details}

The process flow for shallow water acoustic communication is displayed in Figure 6. Low frequency acoustic signals $1 \mathrm{kHz}, 1.5 \mathrm{kHz}$ generated in the computer with MATLAB software are the inputs to the water tank. Those low frequency signals with peak to peak signal strengths of $1 \mathrm{~V}, 1.5 \mathrm{~V}$ and $2 \mathrm{~V}$ are experimented. The generated signal is sent to a generic concealed speaker kept in one end of water tank which transmits the acoustic signal to channel. At the other end of channel concealed microphone picks the communicated signal and received signal is taken up for future analysis. Entire experimental work is taken up for transmission ranges, $1.2 \mathrm{~m}$ and $2.5 \mathrm{~m}$. The experimental and simulation outcomes are compared. 


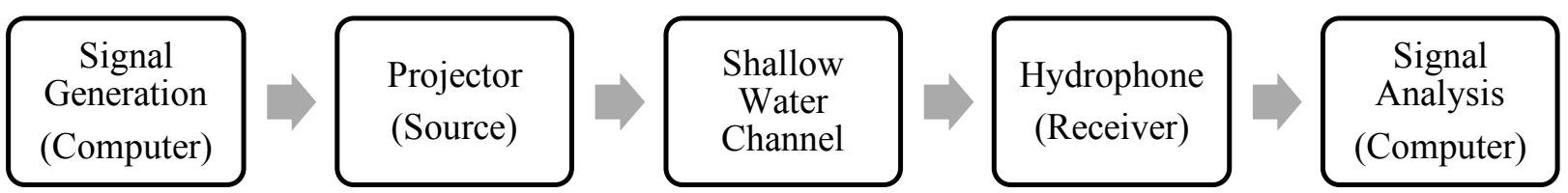

Figure 6. Process flow for acoustic communication in shallow water channel.

\section{Test Results}

Signal recorded for different transmission ranges in the experimental setup displayed in Figure 5(b) are presented and analyzed in this section. The source transmits low frequency acoustic signals $1 \mathrm{KHz}, 1.5 \mathrm{kHz}$ through UTB for various signal strengths of peak to peak $1 \mathrm{~V}, 1.5 \mathrm{~V}$ and $2 \mathrm{~V}$ and they are received at the other end. Table 5 displays the received signal strength at hydrophone (Hyr) and the corresponding attenuation (Att) for transmission range $2.5 \mathrm{~m}$ when acoustic signal of $1 \mathrm{kHz}$ and $1.5 \mathrm{kHz}$ are transmitted to the water tank. Entire measurement when repeated for transmission range $1.2 \mathrm{~m}$ is tabulated in Table 6.

Table 5: Signal Received at Hydrophone for Range $=2.5 \mathrm{~m}$.

\begin{tabular}{|c|c|c|c|c|c|}
\hline \multirow{2}{*}{$\mathrm{I} / \mathrm{P}(\mathrm{V})$} & \multirow{2}{*}{$\operatorname{PRx}(\mathrm{mV})$} & \multicolumn{2}{|c|}{$\mathrm{F}: 1 \mathrm{kHz}$} & \multicolumn{2}{c|}{$\mathrm{F}: 1.5 \mathrm{kHz}$} \\
\cline { 2 - 6 } & & $\operatorname{Hyr}(\mathrm{mV})$ & $\operatorname{Att}(\mathrm{dB})$ & $\operatorname{Hyr}(\mathrm{mV})$ & $\operatorname{Att}(\mathrm{dB})$ \\
\hline 1 & 353.5 & 94.6 & -11.45 & 34.5 & -20.21 \\
\hline 1.5 & 530.3 & 48.9 & -20.70 & 31.6 & -24.49 \\
\hline 2 & 707.1 & 20.5 & -30.75 & 45.7 & -23.79 \\
\hline
\end{tabular}

Table 6: Signal Received at Hydrophone for Range $=1.2 \mathrm{~m}$.

\begin{tabular}{|c|c|c|c|c|c|}
\hline \multirow{2}{*}{$\mathrm{I} / \mathrm{P}(\mathrm{V})$} & \multirow{2}{*}{ PRx $(\mathrm{mV})$} & \multicolumn{2}{|c|}{$\mathrm{F}: 1 \mathrm{kHz}$} & \multicolumn{2}{|c|}{$\mathrm{F}: 1.5 \mathrm{kHz}$} \\
\cline { 3 - 6 } & & $\mathrm{Hyr}(\mathrm{mV})$ & $\operatorname{Att}(\mathrm{dB})$ & $\operatorname{Hyr}(\mathrm{mV})$ & Att(dB) \\
\hline 1 & 353.5 & 49.8 & -16.56 & 96.2 & -11.30 \\
\hline 1.5 & 530.3 & 78.8 & -17.02 & 53.0 & -20.00 \\
\hline 2 & 707.1 & 31.7 & -26.97 & 58.2 & -21.69 \\
\hline
\end{tabular}

$\mathrm{I} / \mathrm{P}-\mathrm{V}_{\mathrm{PP}}$ of Input Signal; PRx $-\mathrm{V}_{\mathrm{rms}}$ of Projector Signal; Hyr $-\mathrm{V}_{\mathrm{rms}}$ of Hydrophone Signal

The experiment results also reflect observation similar to simulation work. That is, lower input signal strength offers high signal reception compared to input signal strengths of higher value. When received signal for two transmission ranges are compared, one more divergence is noticed. With input signal strength of $1 \mathrm{Vpp}$, more received signal strength is observed for transmission range $2.5 \mathrm{~m}$ than $1.2 \mathrm{~m}$ for $1 \mathrm{kHz}$ transmission frequency. Also, in transmission range $2.5 \mathrm{~m}$, acoustic signal frequency $1.5 \mathrm{kHz}$ is attenuated more, whereas, in transmission range $1.2 \mathrm{~m}$, $1 \mathrm{kHz}$ is more attenuated. All the observations show the existence of inconsistency and changeability in shallow water acoustic channel even for a variation of $500 \mathrm{~Hz}$. 


\section{DISCUSSION ON TEST AND SIMULATION RESULTS}

The basic interest of this paper is to bring out the ideal channel behavior for time-invariant shallow water channel in short range. In this research work, received signal estimated by simulation of multipath propagation based on its interaction with channel boundary and measured received signal from shallow water experiments are presented. Experiments were conducted for very close transmission ranges of $1.2 \mathrm{~m}$ and $2.5 \mathrm{~m}$, since multipath occurrence is strongly anticipated. The same is used in simulation also. This brings close comparison between the two so that valuable research findings are nailed out which can guide in building precise model for shallow water acoustic channel.

In simulation results reported for different multipath - channel interaction patterns in Tables 1,2,3 wherever maximum received signal are noticed they are highlighted. No particular multipath pattern showed its dominance. Further, they exhibit conflicting multipath pattern dominance when transmission range, frequency and signal strengths are varied. This reflects the inconsistency in multipath signal interaction patterns with shallow water channel boundary which raise reservation on wide-ranging reflection loss from top sea water surface and drives us to revisit those views. Both simulation and experiment results showed better signal reception for lower input signal strength compared to higher values.

For comparison, average received signal arrived from simulation of various multipath patterns and measurements from experiment for two transmission ranges and two input signal frequencies are tabulated in Tables 7 -10. This provides benchmark on the minimum limit of received signal strength in multipath scenario. Frequency $1.5 \mathrm{kHz}$, offers more signal reception for relatively close transmission range of $1.2 \mathrm{~m}$, whereas for long-range $2.5 \mathrm{~m}$, frequency $1 \mathrm{kHz}$ is received better. However, for various transmission ranges and frequencies, low attenuation is noticed for input signal strength of $1 \mathrm{Vpp}$ from Tables 5-8. The observation presented here gives an idea about the shallow water channel behavior for very close-range acoustic communication.

Table 7: Comparison of Simulation and Experiment Results $-\mathrm{f}: 1 \mathrm{kHz}-$ Range $=2.5 \mathrm{~m}$.

\begin{tabular}{|c|c|c|c|}
\hline $\mathrm{I} / \mathrm{P}(\mathrm{V})$ & PRx $(\mathrm{mV})$ & Simulation: Average of RX (mV) & Experimental (Hyr) (mV) \\
\hline 1 & 353.5 & 93.95 & 94.60 \\
\hline 1.5 & 530.3 & 49.28 & 48.90 \\
\hline 2 & 707.1 & 18.81 & 20.50 \\
\hline
\end{tabular}

Table 8: Comparison of Simulation and Experiment Results - f: $1.5 \mathrm{kHz}-$ Range $=2.5 \mathrm{~m}$.

\begin{tabular}{|c|c|c|c|}
\hline $\mathrm{I} / \mathrm{P}(\mathrm{V})$ & PRx $(\mathrm{mV})$ & Simulation: Average of RX $(\mathrm{mV})$ & Experimental (Hyr) (mV) \\
\hline 1 & 353.5 & 47.96 & 45.70 \\
\hline 1.5 & 530.3 & 35.76 & 34.50 \\
\hline 2 & 707.1 & 34.35 & 31.60 \\
\hline
\end{tabular}


Table 9: Comparison of Simulation and Experiment Results - f: $1 \mathrm{kHz}-$ Range $=1.2 \mathrm{~m}$.

\begin{tabular}{|c|c|c|c|}
\hline $\mathrm{I} / \mathrm{P}(\mathrm{V})$ & PRx $(\mathrm{mV})$ & Simulation: Average of RX $(\mathrm{mV})$ & Experimental $(\mathrm{Hyr})(\mathrm{mV})$ \\
\hline 1 & 353.5 & 78.10 & 78.80 \\
\hline 1.5 & 530.3 & 48.39 & 49.80 \\
\hline 2 & 707.1 & 36.31 & 31.70 \\
\hline
\end{tabular}

Table 10: Comparison of Simulation and Experiment Results - f: $1.5 \mathrm{kHz}$ - Range $1.2 \mathrm{~m}$.

\begin{tabular}{|c|c|c|c|}
\hline $\mathrm{I} / \mathrm{P}(\mathrm{V})$ & PRx $(\mathrm{mV})$ & Simulation: Average of RX $(\mathrm{mV})$ & Experimental $(\mathrm{Hyr})(\mathrm{mV})$ \\
\hline 1 & 353.5 & 95.50 & 96.20 \\
\hline 1.5 & 530.3 & 56.60 & 58.20 \\
\hline 2 & 707.1 & 53.00 & 53.00 \\
\hline
\end{tabular}

\section{CONCLUSION}

In this paper, the simulation of various multipath patterns of signal propagation in shallow water environment and experiment results for short range is presented. The three-path model is formed by signal travel towards the top channel boundary and channel bottom along with direct path. Once the signals interact with channel boundary, they return to the receiver. The eight possible multipath propagation patterns and their influence on the received signal are highlighted here.

In laboratory, repeated transmission of low frequency acoustic signals $1 \mathrm{kHz}$ and $1.5 \mathrm{kHz}$ of various signal strengths is exercised, and received signals are analyzed. The theoretical predictions of certain patterns of multipath signal interaction with channel boundary are close to channel measurements which unveil the promising probable multipath patterns. Also, the nearness of received signal between experiment results and average received signal arrived from the simulation of multipath patterns hints on the range of signal strength required for reception. This information is significant in the design of low power signal recovery circuit for shallow water applications. Both experiment and simulation results reveal that lower input signal strengths are received better compared to higher signal strengths. Overall, inconsistency observed in received signal from all the simulated multipath patterns and experiment operated at varied signal frequency, transmission range and input signal strength exhibit the unpredictable behavior of shallow water channel even in time invariant condition. The extent of signal deterioration presented by time delay infusion in multipath signal travel here drives towards the necessity to revisit generalized multipath channel model for shallow water based on ideal reflection coefficients. The research outcomes of multipath signal propagation strongly find its potential role in applications related to underwater sensor networks where fundamental study on battery life assessment and power utilized at each node is vital.

\section{ACKNOWLEDGMENT}

The authors thank Visvesvaraya PhD Scheme for Electronics and IT of MeitY, Government of India, for awarding $\mathrm{PhD}$ fellowship. 


\section{REFERENCES}

Alenka G. Zajic. (2011). Statistical Modeling of MIMO Mobile-to-Mobile Underwater Channels. IEEE Transaction of Vehicular Technology. 60 (4): 1337 - 1351.

Chitre, M. (2007). A high-frequency warm shallow water acoustic communications channel model and measurements. Journal of Acoustical Society of America. 122:2580-2586.

David Brady \& James C. Preisig. 1998. Underwater Acoustic Communications. In: Poor, H.V \& Wornell, G.W.(Eds.). Wireless Communications: Signal Processing Perspectives. Pp.330-379. Prentice-Hall, Inc, Upper Saddle River.

Fisher,F; \& Simmons,V . (1977). Sound absorption in sea water. The Journal of the Acoustical Society of America, 62:558-564.

Flatte, S.M. 2010. Sound transmission through a fluctuating ocean. Cambridge Monographs on Mechanics, Cambridge University Press, Cambridge.

Hovannes Kulhandjian, Tommaso Melodia. (2014). Modelling Underwater Acoustic Channels in Short-range Shallow Water Environments, in Proceedings of the International Conference on Underwater Networks \& Systems (WUWNET'14), 1-5.

Kaddouri, S; Beaujean, P.-P.J; Bouvet, P.-J. (2018). High frequency acoustic estimation of time-varying underwater sparse channels using multiple sources and receivers operated simultaneously. IEEE Access, 6(1): 10569-10580.

Landolsi, M.A; Muqaibel, A; Almutairi, A. (2016). UKF-based channel estimation and LOS/NLOS classification in UWB wireless networks. Journal of Engineering Research, 4(2):86-102.

Parastoo Qarabaqi; \& Stojanovic, M. (2013). Statistical Characterization and Computationally Efficient Modeling of a Class of Underwater Acoustic Communication Channels. IEEE Journal of Ocean Engineering, 38 (4): 701-717.

Peterson, J.C., \& Porter, M.B. (2013). Ray/beam tracing for modeling the effects of ocean and platform dynamics, IEEE Journal of Ocean Engineering, 38 (4): 655-665.

Stojanovic, M., \& Preisig, J. (2009). Underwater Acoustic Communication Channels: Propagation Models and Statistical Characterization, IEEE Magazine, 47:84-89.

Stojanovic, M. (2006). On the Relationship Between Capacity and Distance in an Underwater Acoustic Communication Channel, in Proceedings of the 1st ACM international workshop on Underwater networks, 41-47.

Stojanovic, M \& Pierre-Philippe Beaujean. 2016. Springer Handbook of Ocean Engineering. Dhanak, Xiros(Ed.). Acoustic Communication. Pp. 359-386. Springer Nature Switzerland.

Thorp, W.H. (1967). Analytic Description of the Low-Frequency Attenuation Coefficient. Acoustical Society of America Journal, 42: 270.

Tri Budi Santoso; EndangWidjiati; Wirawan; Gamantyo Hendrantoro. (2016). The Evaluation of Probe Signals for Impulse Response Measurements in Shallow Water Environment. IEEE Transactions on Instrumentation and Measurement, 65(6):1292-1299.

Umair Mujtaba Qureshi; Faisal Karim Shaikh; Zuneera Aziz; Syed M. Zafi S. Shah; Adil A. Sheikh; Emad Felemban; Saad Bin Qaisar. (2016). RF Path and Absorption Loss Estimation for Underwater Wireless Sensor Networks in Different Water Environments. Sensors, 16(6):890.

Urick, R.J. 1984. Ambient Noise in the sea. Peninsula publishing, California. 
Van Duc Nguyen; Tien Hoa Nguyen, Hoas Xuan Thi HO. (2019). Methods to Estimate the Channel Delay Profile and Doppler Spectrum of Shallow Underwater Acoustic Channels. Archives of Acoustics, 44(2):375383.

Virovlyansky, A.L. (2008). Ray Chaos in Underwater Acoustic Waveguides. International Journal of Bifurcation and Chaos. $18: 2693-2700$.

Wang, X; Wang, X; Jiang, R; Wang, W; Chen, Q; Wang, X. (2019). Channel modelling and estimation for shallow underwater acoustic OFDM communication via simulation platform. Applied Science. 9(3):1-19.

Yang,T.C. (2012). Properties of underwater acoustic communication channels in shallow water, Journal of Acoustical Society of America.131:129-145. 